\title{
Structure Optimization of Plastic Part Based on Moldflow
}

\author{
Jie Wang \\ Engineering Department, \\ Tangshan College, \\ Tangshan, China
}

\begin{abstract}
Product structure and forming scheme of seeder's typical part were analyzed and optimized in this topic, which affecting the warping deformation of plastic part using Moldflow software, ultimately the best solution was determined. The defects of mould design and plastic parts manufacturing injection moulding occur in the process can be effectively avoided using CAE software simulation. This simulation method can conduct mould structure optimization, and reduce repeat mould repair and the waste of mould trial energy, and improve rate of finished product.
\end{abstract} tic part

Keywords-moldflow; optimize; warping deformation; plas-

\section{INTRODUCTION}

The factors affecting the quality of plastic parts include weld mark, warping deformation, shrink index, cavitations, etc [1]. Warping deformation is the most intuitive factor impact to the quality of plastic products [2]. Warping deformation is common defect in the production and design of injection mould. It is also a defect of products difficult to solve [3]. Product structure and forming scheme of seeder's typical part were analyzed and optimized in this topic, which affecting the warping deformation of plastic part using Moldflow software. Ultimately the best solution was determined [4] [5].

\section{Meshing OF PLASTIC PART}

Digitizing and three-dimensional model had been converted formats and imported into Moldflow. Then the model had been meshed. The result of meshing was shown in Figure 1.

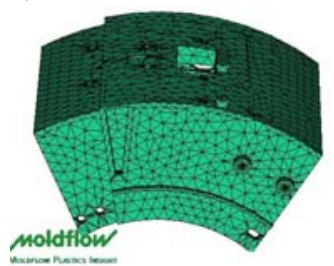

Figure 1. Mesh of plastic part.

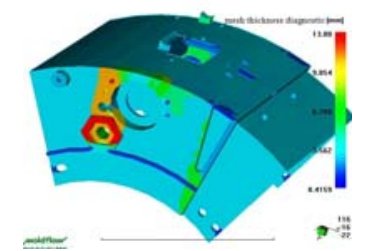

Figure 2. Measuring result of the plastic part thickness.

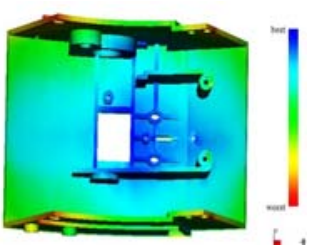

Figure 3. Analysis results of the best gate location.

The thickness of plastic part measuring result was shown in Figure 2. Figure 2 illustrates that most of the thickness of the plastic part was uniform, thickness of parts is bigger in some places. The maximum thickness is $13 \mathrm{~mm}$.

\section{ANALYZING THE EFFECTS OF GATE LOCATION AND NUMBER PREJUDICE ON THE WARPING DEFORMATION}

The effects of the gate location and number prejudice the warping deformation were analyzed through different scheme comparison.

\section{A. Determine the Location and Area of the Best Gate}

The location of the best gate was analyzed before the injection mould casting system was created. The result was shown in Figure 3.

\section{B. Determine the Location and Number of the Gate}

Five schemes of the gate location and number were determined due to the location of the best gate according to the structure and material properties of the plastic part. The best scheme of injection moulding would be determined through comparing the analysis results of several schemes.

Scheme 1 was horizontal rectangle gate with two sides (shown in Figure 4(a)), Schemes 2-4 adopted single rectangular gate (shown in Figure 4(b-d)). Scheme 5 was vertical rectangle gate with two sides (shown in Figure 4(e)). Those schemes were shown in Figure 4. 


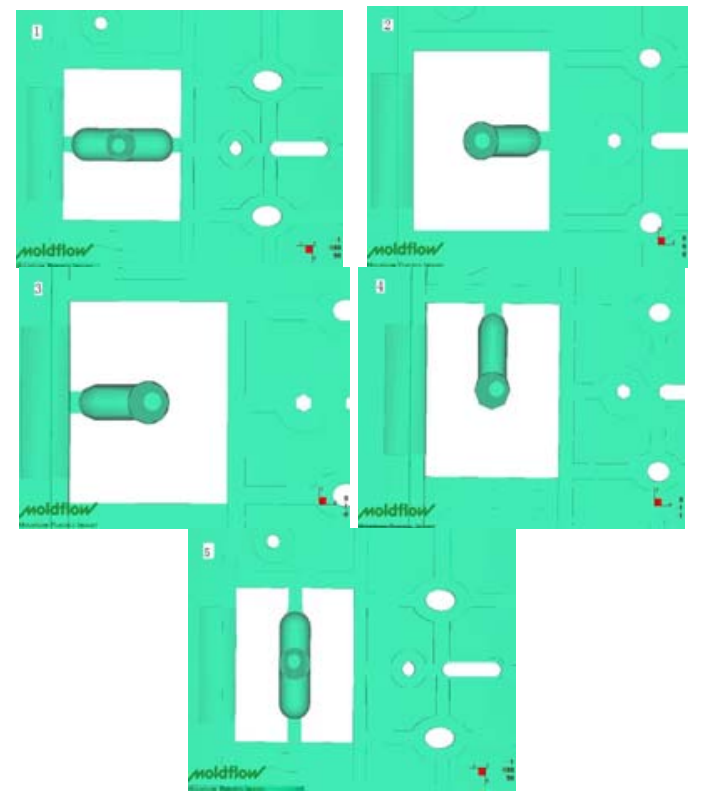

Figure 4. Five kinds of schemes of the gate. (a) Rectangle gate with two sides (horizontal) of scheme 1; (b) Rectangle gate with one side (right) of scheme 2; (c) Rectangle gate with one side (left) of scheme 3; (d) Rectangle gate with one side (upside) of scheme 4 and (e) Rectangle gate with two sides (vertical) of scheme 5 .

\section{Moldflow Analysis}

The Material of the plastic part was ABS. Injection moulding process parameters were set as follows according to system recommendations of $\mathrm{ABS}$, mould temperature was $60^{\circ} \mathrm{C}$, melt temperature was $230^{\circ} \mathrm{C}$. Then dynamic filling process, cooling procedure and warping were simulated. The results were shown in Figure 5.

The results shown that the maximum warping deformation was as follows: 2.852mm (shown in Figure 5(a)), $1.792 \mathrm{~mm}$ (shown in Figure 5(b)), $1.836 \mathrm{~mm}$ (shown in Figure 5(c)), 1.83mm (shown in Figure 5(d)), 1.778mm (shown in Figure 5(e)). This shows that the difference between the minimum and maximum warping deformation was $37.7 \%$, because of the difference in gate location and number with the conditions of simulation agreement. It was shown that the warping distortion of the fifth Scheme was the smallest.

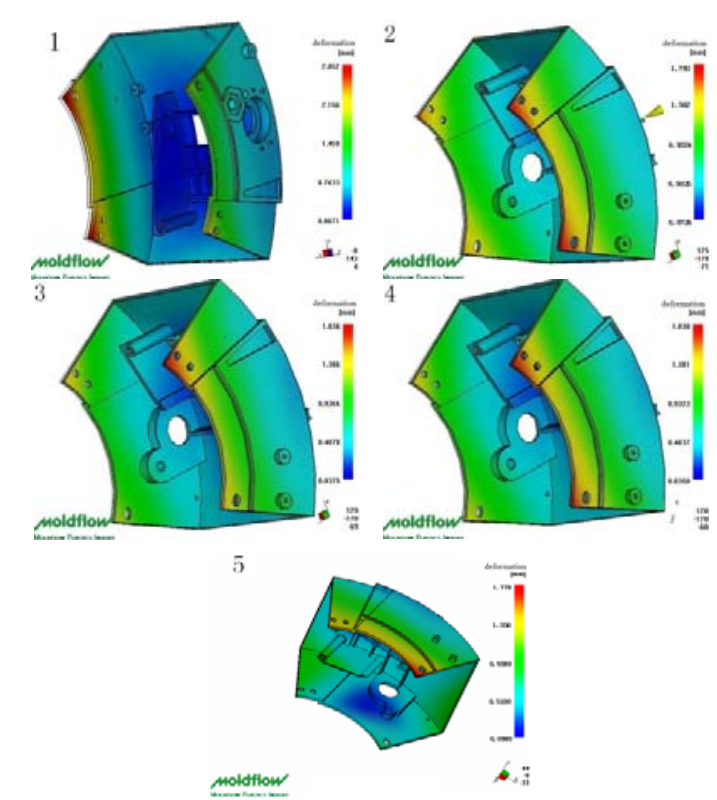

Figure 5. Warping analysis results of five kinds of schemes. (a) Warping deformation of scheme 1; (b) Warping deformation of scheme 2; (c) Warping deformation of scheme 3 ; (d) Warping deformation of scheme 4; (e) Warping deformation of scheme 5.

\section{RESEARCH THE EFFECT ON WARPING DEFORMATION OF NON-UNIFORMITY OF THE PLASTIC PARTS’ WALL THICKNESS}

Taking scheme 1 as an example, analyze the variations of warping deformation before and after uniform the plastic parts' wall thickness. The minimum warping deformation was $2.852 \mathrm{~mm}$ before uniform the plastic parts' wall thickness, it was shown in Figure 5(a). The result of thickness test of scheme 1 was shown in Figure 6. The maximum wall thickness was $6.292 \mathrm{~mm}$ (the maximum wall thickness was $13 \mathrm{~mm}$ before uniform the plastic parts' wall thickness).

The result of Moldflow analysis again were shown in Figure 7(a) and (b). The maximum warping deformation was $1.144 \mathrm{~mm}$.

Warp is the permanent strain of the plane caused by injection moulding instead of load. This phenomenon of warp may occur because of the uneven contraction when the inner stress has been released, which is occurred during injection. Different cooling rates could cause different rates shrink. The warp arises because the cooling rate of the thicker parts of the plastic part is smaller than the thin parts. The result indicated that the maximum warping deformation was changed from $2.852 \mathrm{~mm}$ to $1.144 \mathrm{~mm}$. The maximum warping deformation was decreased by 59.9 percent. Thus, it can be seen that non-uniformity of the plastic part wall thickness has a great effect on the warping deformation so the designers should uniform the plastic part' wall thickness in the process of product design in order to decrease the warping deformation. 


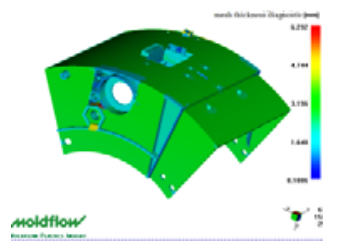

Figure 6. The result of thickness test after uniform the plastic parts' wall thickness.

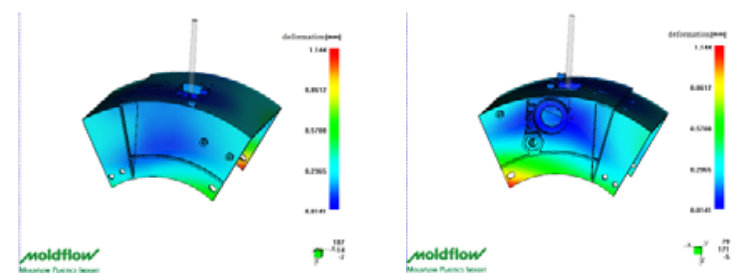

Figure 7. Analysis result views of warping deformation after uniform the plastic parts' wall thickness. (a) the analysis result view1; (b) the analysis result view 2 .
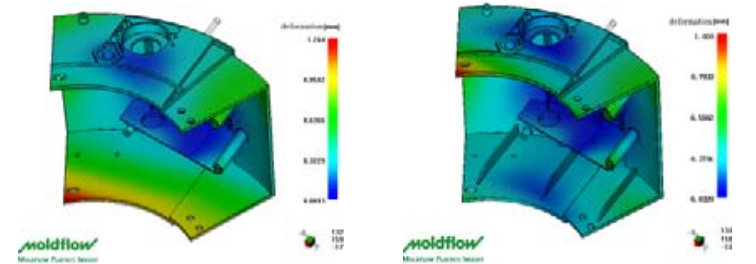

Figure 8. Comparisons of warping deformation before and after adding the strengthening rib. (a) The result of Moldflow analysis without the strengthening rib; (b) The result of Moldflow analysis with the strengthening rib.

\section{DECREASE THE WARPING DEFORMATION USING THE STRENGTHENING RIB}

Taking scheme 2 as an example, the warping deformation could be decreased using the method of adding strengthening rib to the plastic part. The result of Moldflow analysis again was shown in Figure 8(a and b).

Result indicated that the maximum warping deformation was changed from $1.264 \mathrm{~mm}$ to $1.02 \mathrm{~mm}$. The maximum warping deformation was decreased by 19.3 percent.

\section{CONCLUSION}

In this topic, product structure and forming scheme of seeder's typical part were analyzed and optimized, which affecting the warping deformation of plastic part using Moldflow software. The warping deformation could be decreased evidently using the method of adding strengthening rib to the plastic part and uniform the plastic part' wall thickness. The results shown that plastic CAE simulation could effectively eliminate problems in mould design and manufacturing process, and could replace the traditional modes of repeating testing and repairing molds, thereby reduce manufacturing costs and shorten the product development cycle.

\section{REFERENCES}

[1] Wang Jie, Wang Jian-ling, Shang Xing-guo, Bai Fan, Failure prediction of injection moulded plastic part and parameter optimization based on Moldflow. Journal of Manufacturing automation, 11(32), pp. 145-147, 2010.

[2] Zhu Chun-Dong, Yu Wei-hua, Zhu Hong-Yan, Guo Wei, Numerical simulation on the effect of technological parameters on the warpage of plastic thin shelled component. Journal of Die and Mould Industry, 33(9), pp. 50-53, 2007.

[3] Wang Gang, Shan Yan, Application Example of Mold Flow Analysis Based on Moldflow. Tsinghua University Press, Beijing, 2005.

[4] Chen Zhi-Yong, Injection Molding from Entry to the Master Based on Moldflow6.1. Electronic Industry Press, Beijing, 2009.

[5] Heng Sheng-Jie, Mold Development and Analysis of the Whole Process Based on Pro/ENGINEER + Moldflow. China Youth Press, Beijing, 2008. 